\title{
Effects of a Disturbed Kinetic Chain in the Fastball Pitch on Elbow Kinetics and Ball Speed ${ }^{+}$
}

\author{
Ton (A. J. R.) Leenen ${ }^{1, *}$, Bart Van Trigt ${ }^{2}$, Marco (M. J. M.) Hoozemans ${ }^{1}$ \\ and DirkJan (H. E. J.) Veeger ${ }^{2}$ \\ 1 Department of Human Movement Sciences, Faculty of Behavioural and Movement Sciences, \\ Vrije Universiteit Amsterdam, Amsterdam Movement Sciences, van der Boechorstraat 9, \\ 1081 HV Amsterdam, The Netherlands; m.j.m.hoozemans@vu.nl \\ 2 Department of Biomechanical Engineering, Faculty of Mechanical, Maritime and Material Engineering, \\ Delft University of Technology, Mekelweg 2, 2628 CD Delft, The Netherlands; \\ b.vantrigt@tudelft.nl (B.V.T.); h.e.j.veeger@tudelft.nl (D.V.) \\ * Correspondence: a.j.r.leenen@vu.nl; Tel.: +31-20-5983605 \\ + Presented at the 13th conference of the International Sports Engineering Association, Online, \\ 22-26 June 2020.
}

Published: 15 June 2020

\begin{abstract}
The purpose of the present study was to investigate the effect of an experimentally imposed kinetic chain disturbance in baseball pitching on ball speed and elbow kinetics. The experimental design consisted of two (within-subject) conditions. In one condition there was no manipulation (control condition). The other condition involved a manipulation of the kinetic chain by taping the pelvis and trunk. In both conditions, pitchers were instructed to throw fastballs until a minimum of 15 pitches were captured. Inverse dynamic solutions were used calculate the internal elbow moments of six elite youth baseball pitchers. The pitchers that were hampered in throwing fastballs, by the taped pelvis and trunk, showed significant lower ball speeds and peak internal varus moments compared to the pitchers that were allowed to throw without any hindrance.
\end{abstract}

Keywords: overhead throwing 1; motion analysis 2; kinematics 3; kinetic chain 4; baseball 5; disruption 6

\section{Introduction}

One of the most valuable skills in overhand sports, specifically in baseball pitching, is the ability to throw a ball at speeds up to 100 miles per hour. This requires a highly coordinated whole-body motion requiring transfer of kinetic energy from the lower body, pelvis and trunk up to the upper body [1]. This transfer of energy through body segments is often referred to as the kinetic chain [1,2] and requires sequential motions of subsequent body segments [3]. This appears to be a key factor for optimal throwing performance [3].

Movements out of sequence may hamper optimal transfer of kinetic energy due to deficiencies in the kinetic chain, which either result in a reduction in performance or in an additional compensation by the upper body segments to accommodate for the energy loss to maintain performance. This mechanism is referred to as the "catch-up" phenomenon [4]. The upper body segments and joints between them are therefore conceivably more prone to (overuse) injuries [3,5]. It can be expected that a kinetic chain disruption, in addition to a possible performance reduction, also results in upper body compensation that can be deduced from changes in upper body kinetics. 
The purpose of the present study was to investigate the effect of an experimentally imposed kinetic chain disturbance in baseball pitching on ball speed and elbow kinetics in elite youth baseball pitchers.

\section{Materials and Methods}

\subsection{Participants}

A total of 8 baseball pitchers of the Dutch AAA team, aged 15 to 23 years, participated in this study (mean age 17.5 SD 2.4 years; mean pitching experience 10.1 SD 2.0 years; mean body weight $79.2 \mathrm{SD} 8.4 \mathrm{~kg}$; mean body height $1.86 \mathrm{SD} 0.06 \mathrm{~m}$ ). Potential participants had to be free from ongoing injury, pain, or muscle soreness that prevented them from throwing a fastball as they would normally do, and they were excluded if they had previous injuries that might lead to restrictions in the kinetic chain (such as permanent restrictions in the range of motion, for example, as a result of elbow or shoulder surgery). The Faculty of Behavioural and Movement Sciences' local ethics committee approved the study under reference number VCWE-2019-033, and all participants gave their written consent according to the university policy, after being fully informed about the content and purpose of the study.

\subsection{Procedures}

Data collection took place at the movement laboratory of the Royal Netherlands Football Association. Upon arrival, after the objectives and procedures were explained, the pitchers were asked to read and sign the informed consent forms. Participants then changed into tight-fitting trousers or shorts and indoor shoes, whereafter 10 reflective markers (10 $\mathrm{mm}$ in diameter) were attached (Figure 1) directly to the skin, where possible at anatomic bony landmarks, with doublesided tape. The markers were attached according to the plug-in-gait model, extended with upper extremity markers (Figure 1). After the marker setup, participants were given unrestricted time to warm up as they would normally do for a bullpen session, which consisted of running, stretching and a specific throwing session. Subsequently, pitchers performed 5 to 10 submaximal fastball pitches from a custom-made pitching mound to become comfortable with the experimental setup. All pitches had to be aimed at a rectangular strike zone (height $0.64 \mathrm{~m}$; width $0.38 \mathrm{~m}$ ) $0.55 \mathrm{~m}$ above the ground, which was taped on a tensioned net at the regular game distance of $18.4 \mathrm{~m}(60.5 \mathrm{ft})$.

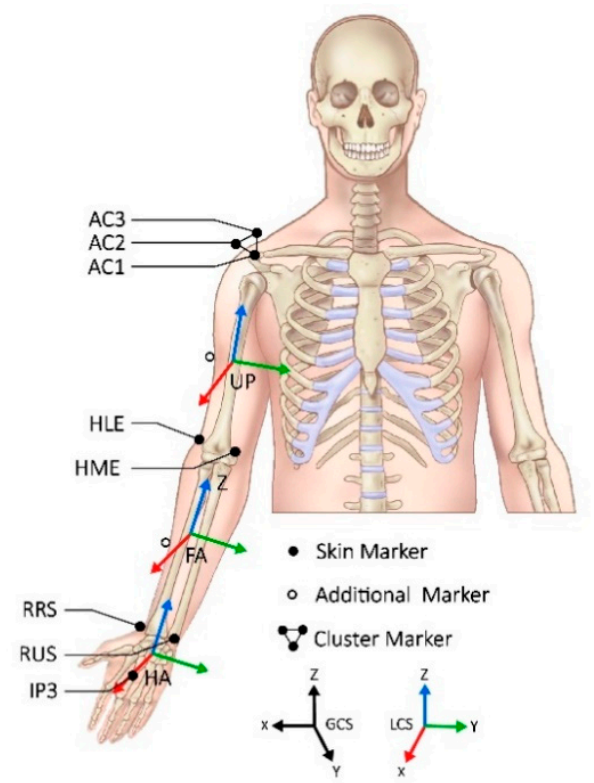

Figure 1. Reflective marker locations, local coordination systems of the upper extremity (HA, Hand; FA, Forearm; UP, Upperarm) and definition of the global coordination system. 
The experimental design consisted of two (within subject) conditions. In one condition there was no manipulation (control condition). The other condition involved a manipulation of the kinetic chain (Figure 2), which was applied between the pelvis and trunk segments, as the relatively high mass of the trunk segment is designated as one of the key contributors to the energy development and transference in the kinetic chain [6-8]. The sequence of the two conditions presented to each subject was randomly balanced between subjects.

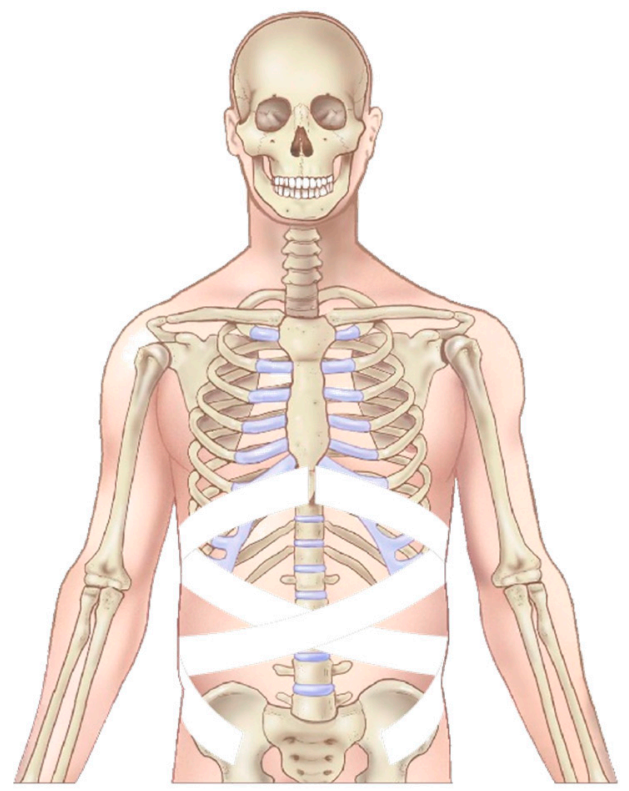

Figure 2. Manipulation of the kinetic chain by attaching four strokes of short-stretch (60\%) tape in a circular manner at the skin around the lower part of the trunk of the participant from the pelvis to the trunk at the height of sternum.

In both conditions, pitchers were instructed to throw fastballs as fast and accurately as possible to the strike zone. Pitches were considered qualified for analysis if the backstop was hit. Each participant continued to throw fastballs until a minimum of 15 qualified pitches were captured. The participants, on average, performed 25 throws before the requirements were met. Pitches were selected for data analysis based on the quality of the kinematic data.

\subsection{Data Acquisition}

Kinematic data were captured using an 8-camera motion analysis system (model V5; Vicon Motion Systems Ltd., Yarnton, UK) with Vicon Nexus automatic digitization software (version 2.7; Vicon Motion System Ltd., Yarnton, UK). The 3D marker positions were captured at 400 frames/s. A radar gun (model Stalker Pro II Sport; Applied Concepts Inc., Plano, TX, USA) was used to capture the ball speed of each throw directly before the rectangular strike zone was hit.

\subsection{Data Analysis}

Position data of markers were exported from Vicon Nexus software [6], and all subsequent data processing and reductions were conducted with MATLAB software R2019a, update 3, version 9.6 (The MathWorks, Inc., Natick, MA, USA). The 3D positional time series of the markers were splined with the standard cubic spline interpolation function available in MATLAB to account for missing data and filtered with a low-pass fourth-order zero-lag Butterworth filter using an estimated optimal cut-off frequency of $17 \mathrm{~Hz}$ to reduce the effects of sampling error [9]. The anatomical coordinate systems of the hand and forearm segments and the elbow joint were defined according to the recommendations of the International Society of Biomechanics (ISB), except that anatomical coordination systems were rotated 90 degrees clockwise over the $x$-axis [10,11] (Figure 1). The global coordinate system was defined with the (positive) $\mathrm{y}$-axis in the throwing direction, the $\mathrm{z}$-axis pointing in the upward direction, and the $\mathrm{x}$ - 
axis pointing to the right, according to the right-hand rule (Figure 1). The internal net joint forces and moments at the elbow joint were calculated by means of inverse dynamic solutions [12]. The internal net forces and moments at the elbow joints were calculated as the forces and moments of the proximal segment acting on the distal segment, according to Hof [13]. The segment mass, location of the center of mass, and moment of inertia were estimated based on Zatsiorsky [14].

The event of ball release was modelled by linearly decreasing the ball mass, assuming that the center of mass of the hand and ball overlap, from $100 \%$ to $0 \%$ during $20 \mathrm{~ms}$ before actual ball release [15].

\subsection{Statistical Analysis}

Statistical analysis was conducted in IBM SPSS, version 26.0.0.0 (IBM Corporation, Armonk, NY, USA). Generalized Estimating Equations (GEE) using the "exchangeable" working correlation structure were used to identify differences between the non-manipulated and manipulated conditions for ball speed and peak elbow moments. The general linear regression equation was used:

$$
\text { outcome }=b_{0}+b_{1} * \text { predictor, }
$$

The participants were considered as a random factor in these analyses to account for the dependency between the repeated throws within participants. Therefore, GEE analysis provides valid standard errors of the parameter estimates regardless of the distribution [16]. The qualitative approved throws per condition per participant were used for statistical analysis. The conditions (taped pelvis and trunk and control) were added to the model as categorical predictors (factors), while ball speed ( $\mathrm{mph}$ and $\mathrm{m} / \mathrm{s}$ ) and elbow moments $(\mathrm{Nm})$ were added as the continuous outcomes. The predictors' regression coefficients $\left(b_{1}\right)$ and corresponding $95 \%$ confidence intervals (CI) were determined by the Wald chi-square statistic. An a-priori $\alpha$ level of 0.05 was used to determine statistical significance.

\section{Results}

Preliminary results are presented here. Two pitchers were excluded from biomechanical analysis due to missing indispensable markers from the throwing arm.

\subsection{Ball Speed}

The average ball speed of fastball pitches was statistically significant lower in the condition where the kinetic chain was manipulated compared to the condition where the manipulation was absent by $0.5 \mathrm{mph}, 95 \%$ Wald CI [-0.05, -0.9$]$ or $0.3 \mathrm{~m} / \mathrm{s}, 95 \%$ Wald CI [-0.4, -0.02$]$ (Table 1 ).

Table 1. Ball speed for the conditions normal pitching and pitching with taped pelvis and trunk estimated with GEE $(\mathrm{N}=8)$.

\begin{tabular}{cccccc}
\hline & \multicolumn{5}{c}{ Conditions } \\
\cline { 2 - 6 } & \multicolumn{2}{c}{ Normal Pitching } & \multicolumn{2}{c}{ Pitching with Taped Pelvis and Trunk } & $p$-Value \\
\hline Variable & Estimated Mean & $\mathbf{9 5 \%} \mathbf{C I}^{\mathbf{1}}$ & Estimated Mean & $\mathbf{9 5 \% \mathbf { C I } ^ { \mathbf { 1 } }}$ \\
\hline Ball speed (mph) & 76.9 & 74.8 to 78.9 & 76.4 & 74.4 to 78.3 & $0.028^{2}$ \\
Ball speed (m/s) & 34.4 & 33.4 to 35.3 & 34.1 & 33.3 to 35.0 & $0.028^{2}$ \\
\hline
\end{tabular}

${ }_{1}^{1} 95 \%$ Wald Confidence Interval; ${ }^{2}$ Statistically significant $(\alpha=0.05)$.

\subsection{Elbow Moments}

Peak internal varus moment was $5.5 \mathrm{Nm}$ (95\% Wald CI [0.8, 10.3]) lower when pitchers were taped while throwing fastballs compared to the condition with no tape (Table 2). No other significant differences in peak elbow moments were found between normal pitching and the condition where the kinetic chain was disrupted by taping the pelvis and trunk. 
Table 2. Peak internal net elbow moments for the conditions of normal pitching and pitching with taped pelvis and trunk estimated with GEE $(\mathrm{N}=6)$.

\begin{tabular}{|c|c|c|c|c|c|}
\hline \multirow[b]{3}{*}{ Variable } & \multicolumn{4}{|c|}{ Conditions } & \multirow{3}{*}{$p$-Value } \\
\hline & \multicolumn{2}{|c|}{ Normal Pitching } & \multicolumn{2}{|c|}{ Pitching with Taped Pelvis and Trunk } & \\
\hline & Estimated Mean & $95 \% \mathrm{CI}^{1}$ & Estimated Mean & $95 \% \mathrm{CI}^{1}$ & \\
\hline Peak Varus Moment (Nm) & 64.2 & 58.9 to 69.6 & 58.7 & 54.0 to 63.3 & $0.0222^{2}$ \\
\hline Peak Valgus Moment (Nm) & -37.5 & -50.3 to -24.6 & -33.0 & -44.8 to -21.3 & 0.122 \\
\hline Peak Flexion Moment (Nm) & 54.5 & 42.1 to 66.9 & 50.6 & 38.8 to 62.5 & 0.102 \\
\hline Peak Extension Moment (Nm) & -19.0 & -21.8 to -16.3 & -16.7 & -21.4 to -11.9 & 0.113 \\
\hline Peak Pronation Moment (Nm) & 10.2 & 6.5 to 13.9 & 9.7 & 6.5 to 12.8 & 0.254 \\
\hline Peak Supination Moment (Nm) & -4.8 & -6.6 to -3.1 & -5.3 & -7.5 to -3.1 & 0.094 \\
\hline
\end{tabular}

1 95\% Wald Confidence Interval; ${ }^{2}$ Statistically significant $(\alpha=0.05)$.

\section{Discussion}

The aim of this study was to investigate the effect of an experimentally imposed kinetic chain disturbance on ball speed and elbow kinetics in the baseball pitch. The pitchers that were hampered in throwing fastballs, by the taped pelvis and trunk, showed lower ball speeds and peak internal varus moments compared to the pitchers that were allowed to throw without any hindrance. The moments of the elbow in the other directions were equivalent between conditions.

Our hypothesis that pitchers who were hampered in throwing fastballs would exhibit increased internal elbow moments accompanied by a constant or decreased ball speed was not supported by the results of this study. On the contrary, pitchers who were hindered by the taped pelvis and trunk demonstrated decreased ball speed accompanied with decreased elbow moments and a significant decreased internal varus moment in particular. A possible explanation for the non-appearance of the "catch-up" phenomenon in this study was the absence of the urge to perform. Pitchers may be more likely to use upper body compensation strategies when they will be challenged to continue to perform at all times (i.e., retention of ball speed after plurality of repetitions). Another possible explanation is the type of manipulation used to disturb the kinetic chain since it is questionable whether the type of manipulation actually influences the kinetic chain. The time interval between the peak angular velocities of the pelvis and trunk, referred to as the separation time, provides a clear indication of kinetic chain $[17,18]$. Evaluation of the separation time is therefore indispensable to be able to verify whether the taping the pelvis and trunk actually influenced the kinetic chain.

To understand the effect of the kinetic chain on throwing performance and elbow kinetics, further analysis of the timing of pelvis and trunk angular velocities is required. The relationships between the ball speed, elbow kinetics and the timing of the pelvis and trunk angular velocities have already been demonstrated in several studies. The positive association between ball speed and the separation time was demonstrated by a recent study of van der Graaff et al. [17]. A study conducted by Wight et al. demonstrated that pitchers who reach maximum pelvis angular velocity later in the pitch cycle ( $10.8 \%$ vs $26.7 \%$ of the pitch) experienced higher elbow kinetics compared to pitchers who reached their maximum earlier [19]. Aguinaldo et al. reported that pitchers who initiated their trunk rotation before stride-foot contact experienced higher internal elbow varus moments compared to pitchers who did not [20]. The results of these studies underline the necessity to further analyze the timing of the angular velocities of the pelvis and trunk and the conceivable relationship with throwing performance and the kinetic characteristics, which are often attributed as an indicator of stress on the elbow during overhead throwing.

\section{Conclusions}

Pitchers that were hampered in throwing fastballs by disturbing the kinetic chain exhibited decreased ball speed and internal elbow varus moment compared to a condition without any disturbance. Further analysis of the timing of the angular velocities in relation to changes in ball speed and elbow moments are required to further explain the differences found in this study. 
Acknowledgments: This work was supported by the NWO Domain Applied and Engineering Sciences (AES) under project number [R/003635]. This NWO-funded project, named Breaking the High Load-Bad Coordination Multiplier in Overhead Sports Injuries part of the Citius Altius Sanius perspective program (Project 7), is a cooperative effort between the Royal Netherlands Baseball and Softball Federation, Vrije Universiteit Amsterdam, Delft University of Technology, Manual Fysion and PLUX.

Conflicts of Interest: The authors declare no conflict of interest.

\section{References}

1. Putnam, C. Sequential motions of body segments in striking and throwing skills: Descriptions and explanations. J. Biomech. 1993, 26 (Suppl. 1), 125-135.

2. Calabrese, G.J. Pitching mechanics, revisited. Int. J. Sports Phys. Ther. 2013, 8, 652-660.

3. Aguinaldo, A.; Escamilla, R.F. Segmental Power Analysis of Sequential Body Motion and Elbow Valgus Loading During Baseball Pitching: Comparison between Professional and High School Baseball Players. Orthop. J. Sports Med. 2019, 7, doi:10.1177/2325967119827924.

4. van der Hoeven, H.; Kibler, W. Shoulder injuries in tennis players. Br. J. Sport Med. 2006, 40, 435-440, discussion 440, doi:10.1136/bjsm.2005.023218.

5. Oyama, S.; Yu, B.; Blackburn, T.J.; Padua, D.A.; Li, L.; Myers, J.B. Improper trunk rotation sequence is associated with increased maximal shoulder external rotation angle and shoulder joint force in high school baseball pitchers. Am. J. Sports Med. 2014, 42, 2089-2094, doi:10.1177/0363546514536871.

6. Chu, S.K.; Jayabalan, P.; Kibler, B.W.; Press, J. The Kinetic Chain Revisited: New Concepts on Throwing Mechanics and Injury. PMER 2016, 8, S69-S77, doi:10.1016/j.pmrj.2015.11.015.

7. Seroyer, S.T.; Nho, S.J.; Bach, B.R.; Bush-Joseph, C.A.; Nicholson, G.P.; Romeo, A.A. The kinetic chain in overhand pitching: Its potential role for performance enhancement and injury prevention. Sports Health 2010, 2, 135-146, doi:10.1177/1941738110362656.

8. Kibler, B.W.; Sciascia, A. The role of the scapula in preventing and treating shoulder instability. Knee Surg. Sports Traumatol. Arthrosc. 2016, 24, 390-397, doi:10.1007/s00167-015-3736-z.

9. Yu, B.; Hay, J.G. Angular Momentum and Performance in the Triple Jump: A Cross-Sectional Analysis. J. Appl. Biomech. 2015, 11, 81-102, doi:10.1123/jab.11.1.81.

10. Wu, G.; Siegler, S.; Allard, P.; Kirtley, C.; Leardini, A.; Rosenbaum, D.; Whittle, M.; D'Lima, D.; Cristofolini, L.; Witte, H.; et al. ISB recommendation on definitions of joint coordinate system of various joints for the reporting of human joint motion-Part I: Ankle, hip, and spine. J. Biomech. 2002, 35, 543-548, doi:10.1016/s0021-9290(01)00222-6.

11. Wu, G.; Van der Helm, F.C.; Veeger, H.D.; Makhsous, M.; Van Roy, P.; Anglin, C.; Nagels, J.; Karduna, A.R.; McQuade, K.; Wang, X.; et al. ISB recommendation on definitions of joint coordinate systems of various joints for the reporting of human joint motion - Part II: Shoulder, elbow, wrist and hand. J. Biomech. 2005, 38, 981-992, doi:10.1016/j.jbiomech.2004.05.042 .

12. Robertson, D.; Caldwell, G.E.; Hamill, J.; Kamen, G.; Whittlesey, S.N. Research Methods in Biomechanics, 2nd ed.; Human kinetics: Stanningley, 2013; p. 415.

13. Hof, A. An explicit expression for the moment in multibody systems. J. Biomech. 1992, 25, 1209-1211, doi:10.1016/0021-9290(92)90076-d.

14. Zatsiorsky, V.M. Kinetics of Human Motion; Human Kinetics: Stanningley, 2002.

15. Gasparutto, X.; van der Graaff, E.; van der Helm, F.; Veeger, D. Elite Athlete Motor and Loading Actions on The Upper Limb in Baseball Pitching. Procedia Eng. 2016, 147, 181-185, doi:10.1016/j.proeng.2016.06.210.

16. Liang, K.; Zeger, S. Regression Analysis for Correlated Data. Annu. Rev. Publ. Health. 1993, 14, 43-68, doi:10.1146/annurev.pu.14.050193.000355.

17. van der Graaff, E.; Hoozemans, M.; Nijhoff, M.; Davidson, M.; Hoezen, M.; Veeger, D. Timing of peak pelvis and thorax rotation velocity in baseball pitching. J. Phys. Fit. Sports Med. 2018, 7, 269-277, doi:10.7600/jpfsm.7.269.

18. Sgroi, T.; Chalmers, P.N.; Riff, A.J.; Lesniak, M.; Sayegh, E.T.; Wimmer, M.A.; Verma, N.N.; Cole, B.J.; Romeo, A.A. Predictors of throwing velocity in youth and adolescent pitchers. J. Shoulder Elb. Surg. 2015, 24, 1339-1345, doi:10.1016/j.jse.2015.02.015. 
19. Wight, J.; Richards, J.; Hall, S. Influence of pelvis rotation styles on baseball pitching mechanics. Sport Biomech. 2004, 3, 67-83, doi:10.1080/14763140408522831.

20. Aguinaldo, A.L.; Chambers, H. Correlation of throwing mechanics with elbow valgus load in adult baseball pitchers. Am. J. Sports Med. 2009, 37, 2043-2048, doi:10.1177/0363546509336721.

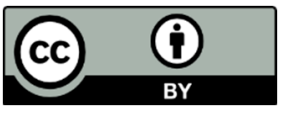

(C) 2020 by the authors. Licensee MDPI, Basel, Switzerland. This article is an open access article distributed under the terms and conditions of the Creative Commons Attribution (CC BY) license (http://creativecommons.org/licenses/by/4.0/). 